\title{
Évaluation moderne, évaluation modeste? Le cas des pôles de compétitivité français
}

\author{
>Émilie-Pauline Gallié ${ }^{a}$ Anna Glaser ${ }^{b}$, \\ Philippe Lefebvre ' et Frédérique Pallez ${ }^{d}$
}

a Chargée de recherche, IMRI, Université Paris Dauphine, PI. Maréchal de Lattre de Tassigny - 75775 Paris Cedex 16

${ }^{b}$ Doctorante, ESCP Europe \& Mines ParisTech 79, avenue de la République - 75543 Paris Cedex 11

'Maître-assistant, Mines ParisTech - 60, boulevard Saint-Michel - 75272 Paris Cedex 06

d Professeur, Mines ParisTech - 60, boulevard Saint-Michel - 75272 Paris Cedex 06

\section{Résumé}

L'évaluation des politiques publiques est un thème récurrent pour les praticiens et chercheurs travaillant sur l'action publique. Mais au-delà de l'accord sur le principe, il n'y a pas stabilisation de la doctrine et de ses pratiques. Différents modèles coexistent ou se succèdent, selon les pays ou les époques. En nous appuyant sur le cas de l'évaluation de la politique française des pôles de compétitivité en 2008, nous montrons les principales difficultés de la démarche, liées au caractère systémique, multi-acteurs et hétérogène de l'objet « cluster ». À travers I'analyse des utilisations de l'évaluation, nous mettons ensuite en évidence que cette évaluation participe à la constitution progressive et simultanée d'une doctrine des clusters et de leur pilotage. En conclusion, nous traçons les bases d'un modèle de démarche évaluative, qui la désacralise et l'intègre à un dispositif plus large de pilotage des politiques publiques.

(c) 2012 IDMP/Lavoisier SAS. Tous droits réservés

Mots clés : cluster, évaluation, politiques publiques, pôle de compétitivité.

\section{Abstract}

What should be expected from a public policy evaluation? The case of the French cluster policy. Public policy evaluations constitute a current topic among practitioners and academics working on public action. However, beyond a mutual agreement on the principles of evaluation, there is hardly any stabilisation of evaluation doctrines and

*Auteur correspondant : frederique.pallez@mines-paristech.fr doi:10.3166/pmp.29.573-590 @ 2012 IDMP/Lavoisier SAS. Tous droits réservés 
practices. Different models coexist, according to the country and the period. Basing our analysis on the evaluation of the French cluster policy in 2008, we highlight the main difficulties of the evaluation process, which are linked to the systemic, multi-actor and heterogeneous character of a "cluster". Through the analysis of how the results of the evaluation are used, we then show that this evaluation takes part of a progressive construction of doctrine on what a cluster is and how it should be managed. In conclusion, we build the basis of an evaluation framework, in which evaluation is integrated in a larger public policy management process.

(c) 2012 IDMP/Lavoisier SAS. Tous droits réservés

Keywords: cluster, competitiveness pole, evaluation, Public policies.

\section{Introduction}

L'évaluation des politiques publiques est, comme la réforme de l'État, une des thématiques récurrentes en France depuis plus de 30 ans dans les débats des praticiens et des chercheurs intéressés par le management public (Trosa, 2009). Chacun appelle de ses vœux l'expansion d'une «culture de l'évaluation ». L'évaluation est devenue un «badge de modernité » selon l'expression de Pollitt (citée par Perret, 2001). Le principe de l'évaluation des politiques publiques est maintenant d'autant moins contesté qu'il est inscrit dans la Constitution depuis juillet 2008 (article 47-2). L'Assemblée nationale devrait même consacrer en principe une semaine par mois au contrôle et à l'évaluation des activités gouvernementales ou des politiques publiques. Néanmoins, les divergences et les doutes apparaissent dès que l'on essaie de préciser les conceptions qui président à l'évaluation, les méthodes mises en œuvre, et les impacts qu'elle peut avoir sur les décisions (Perret, 2001, 2008; Stame, 2009; Thoenig, 2002).

Une occasion d'analyser une évaluation de politique publique « en actes » s'est présentée récemment. En effet, le gouvernement français a lancé en 2005 une politique ambitieuse de clusters, appelés « pôles de compétitivité » ${ }^{1}$. Cette politique est destinée à « renforcer la compétitivité de l'économie française et développer la croissance et l'emploi sur des marchés porteurs $»^{2}$ principalement à travers le soutien financier à des projets de R\&D innovants et collaboratifs entre entreprises et laboratoires de recherche. Dans ce cadre, 71 pôles de compétitivité, répartis sur tout le territoire français, ont été labellisés en 2005. Derrière ce mot de «pôles de compétitivité » se cachent donc des structures regroupant des entreprises et des laboratoires sur des territoires, avec une organisation et une gouvernance dédiées pour susciter les projets et animer l'action collective. Tous ces pôles de compétitivité ont été évalués en 2008 (soit trois ans après leur démarrage) dans le cadre d'une opération d'envergure commanditée par les pouvoirs publics en charge de leur tutelle.

\footnotetext{
1 dont la logique, en tant que politique industrielle, est analysée dans (Menu, 2011).

2 Pour plus d'information sur les pôles de compétitivité, on peut visiter le site officiel : http://competitivite. gouv.fr/
} 
Travaillant depuis 2007 sur les pôles de compétitivité au sein d'une équipe de recherche pluridisciplinaire ${ }^{3}$, nous avons suivi avec attention le déroulement, les résultats et les effets de l'évaluation nationale réalisée en 2008 sur cette politique. Par ailleurs, nous avons pu avoir accès à une partie du matériau brut rassemblé par les évaluateurs, et recueillir les opinions de différents acteurs (notamment dirigeants de pôles) sur cette démarche d'évaluation. À travers ce cas particulier, il est intéressant de remettre en perspective le processus et les résultats de cette évaluation par rapport aux débats contemporains sur l'évaluation, et de tracer, au moins pour les politiques de clusters, les lignes de force des évaluations à venir. En quelque sorte, faire l'évaluation de l'évaluation.

Après avoir rappelé succinctement les grandes problématiques de l'évaluation des politiques publiques, telles qu'elles apparaissent dans une abondante littérature, nous nous focalisons sur la manière dont cette question se décline en pratique sur les politiques de clusters. Suite à cette revue de la littérature, nous analysons les principes et les résultats de l'évaluation des pôles de compétitivité, clusters « à la française ». Nous abordons ensuite les effets de cette évaluation et sa place dans le dispositif de pilotage, pour finalement proposer des pistes sur la manière dont on peut continuer à apprendre de cette opération pour améliorer ses prochaines moutures ${ }^{4}$. En conclusion, nous réinterrogeons la relation entre évaluation et politique publique.

\section{L'évaluation de politiques publiques dans la littérature : un changement de paradigme?}

Il n'est pas dans notre propos de refaire de manière détaillée un panorama de l'histoire de l'évaluation de politiques publiques en France (Thoenig, 2002), ni une discussion des présupposés théoriques qui président aux diverses conceptions de l'évaluation (Chanut, 2009; Stame, 2009). On se contentera de noter qu'en France, après une importation des pratiques américaines par des chercheurs dans les années 70, l'évaluation se développe timidement, avant d'être institutionnalisée en 1990 par un décret qui n'en assure pas, pour autant, l'assise définitive. Après une refonte du système institutionnel en 1998, et une nouvelle mise en sommeil, l'entrée récente du principe dans la Constitution (article 47-2, juillet 2008) pourrait marquer une nouvelle phase de cette institutionnalisation, qui, en France, est considérée comme la clé d'un développement de la pratique évaluative.

Chaque pays a des traditions propres en matière d'évaluation (Bachtler \& Wren, 2006). Ainsi, on sait que dans les pays anglo-saxons, où le rôle de l'État est moins marqué, et où n'existe pas de tradition d'expertise étatique liée à la présence des grands corps techniques, l'évaluation repose davantage sur l'existence d'un corps de professionnels autonomes (Perret 2003). En France, alors que la pratique de l'évaluation de politiques publiques s'est bien établie au niveau régional (grâce par exemple aux évaluations des politiques ou des « programmes » de développement régional de la Commission Européenne), elle peine à se routiniser et se banaliser au niveau étatique. Même si un certain nombre d'évaluations

\footnotetext{
3 Les auteurs appartiennent à une équipe de chercheurs en gestion et en économie qui ont lancé un programme de recherche pluriannuel sur les clusters, financé par l'ANR.

4 Une deuxième évaluation nationale des pôles de compétitivité vient de se dérouler, en 2012.
} 
ont d'ores et déjà été effectuées ${ }^{5}$, de nombreux observateurs sont néanmoins critiques sur l'ampleur et la portée de cette pratique. Il est vrai que toute démarche évaluative se heurte à des problèmes génériques qui dépassent les spécificités nationales.

\subsection{Les difficultés intrinsèques de l'évaluation des politiques publiques}

L'évaluation d'une politique publique consiste à « apprécier son efficacité en comparant ses résultats aux objectifs assignés et aux moyens mis en œuvre » (décret du 18/11/1998). Au-delà de cette définition, apparemment claire, apparaissent au moins trois séries de difficultés, ou de dilemmes, largement connus, que nous nous contentons de rappeler, et qui nous donneront une grille d'analyse de notre cas :

\section{La formulation des objectifs est difficile}

Le travail des évaluateurs consiste souvent à énoncer eux-mêmes les questionnements pertinents sur une politique. Les « résultats », quant à eux, à supposer qu'ils puissent être mesurés ou appréciés, doivent ensuite être intégrés dans une « théorie de l'action » qui en permette l'interprétation (Gibert, 2003). Cependant, la « théorie de l'action » est elle-même objet de débat dans une évaluation, d'autant qu'elle n'a pas nécessairement été formulée lors de la conception de la politique. Certains auteurs (Pawson, 2006; Weiss, 1997), s'appuyant sur le paradigme de la rationalité limitée, sont même allés jusqu'à contester cette vision, qui présuppose la validité de la notion même de « résultats », et doutent que l'on puisse faire autre chose que d'identifier les mécanismes de fonctionnement de systèmes complexes, partiellement autonomes par rapport aux intentions des politiques.

\section{Les choix méthodologiques de la démarche sont problématiques}

Parmi les plus structurantes, trois catégories de questions peuvent être isolées :

- Le moment : Les évaluations peuvent être effectuées à différents moments du déroulement d'une politique, - ex ante, ex post, ex itinere -, ce qui en modifie considérablement le statut et l'usage possible, voire la pertinence (Gibert, 2003). Implicitement, derrière ces options, sont présents des choix et des contraintes variés, en particulier politiques.

- La méthode : Les méthodes utilisées, pour rigoureuses qu'elles cherchent à être, ne peuvent se limiter à des approches purement quantitatives, fondées sur un positivisme étroit. Elles puisent donc dans les méthodes et les grilles des sciences sociales, au prix d'une fragilité apparente plus grande des conclusions. Quel équilibre trouver entre méthodes quantitatives et qualitatives?

- Les participants : La participation des diverses parties prenantes, notamment les usagers ou les bénéficiaires finaux, est toujours source de connaissance. Cependant, l'évaluation ne peut, pour autant, du moins dans l'optique actuelle des pouvoirs publics français, se transformer en instance de négociation, et les conclusions être l'expression des compromis qui en résulteraient. Comment concilier les deux objectifs? D'autant que

5 Des dizaines d'évaluations ont été réalisées depuis 1990, dans le cadre des processus institutionnels mentionnés plus haut, par exemple : les politiques de contractualisation avec les universités, de sécurité routière, de la ville... Par ailleurs, un séminaire commun au cercle de la Réforme de l'État et à la Société Française d'évaluation, démarré début 2010, passe en revue toute une série de démarches évaluatives récentes, qui montrent que cette pratique est assez répandue, quoi qu'on en dise, même si elle est réalisée dans des cadres institutionnels variés. C'est ainsi que la Cour des Comptes a publié en mai 2010 son évaluation de la politique éducative. 
cette vision encore restrictive de l'intervention des parties prenantes est actuellement dépassée ${ }^{6}$ par les partisans d'une évaluation « de $4^{\text {e }}$ génération » (Guba \& Lincoln, 1989), voire « de $5^{\text {e }}$ génération », ou d'une évaluation « émancipatrice » (« empowerment evaluation ») (Fetterman, Kaftarian, \& Wandermans, 1995), permettant à des groupes de citoyens de s'exprimer et d'intervenir directement sur la construction des politiques, donc source de démocratie.

L'interaction entre une évaluation et les suites qui y sont données par les « décideurs » est un phénomène complexe.

Après une période d'attente déçue de la part des tenants de la démarche évaluative, force a été de constater qu' on ne pouvait la considérer, loin de là, comme seule source de la décision politique ou administrative (Lacasse, 1995). De fait, on observe peu d'infléchissements d'une politique liés à un processus évaluatif, et ses effets, sans être inexistants, sont plus diffus et indirects. En effet, au-delà du rôle d'aide à la décision et au pilotage des systèmes de gestion que peuvent jouer les évaluations, ont été mis en évidence des effets d'apprentissage ou de mobilisation des acteurs, ainsi que de déplacement et de partage de représentations. Ces derniers effets se révèlent essentiels dans un contexte où les politiques publiques reposent sur des partenariats entre de nombreux acteurs, et où « la production des normes de l'action publique est partiellement déléguée aux acteurs et parties prenantes » (Perret, 2008).

\subsection{L'évaluation des politiques de clusters : des modèles qui se cherchent}

Ces difficultés d'évaluation des politiques publiques se retrouvent amplifiées en matière d'évaluation des politiques de clusters et de politiques d'innovation en général, alors même qu'est soulignée l'importance de l'évaluation pour l'apprentissage et la transmission du savoir (Potter, 2005). De nombreux travaux existent, tant en France qu'à l'étranger, sur les politiques de clusters, mais bien peu de travaux ont été menés sur l'évaluation de ces politiques (Solvell, 2009). Les auteurs qui s’y sont intéressés (Bellandi \& Caloffi, 2010; Diez, 2001 ; Russo \& Rossi, 2009; Zabala-Iturriagagoitia, Voigt, Gutiérrez-Gracia, \& Jiménez-Saez, 2007) soulignent en particulier la difficulté à spécifier des objectifs souvent impalpables (comme les processus d'apprentissage collectif), la complexité des modèles de causalité, le caractère systémique et dynamique de l'objet à évaluer. La plupart en déduisent la nécessité d'approches nouvelles (sans forcément les spécifier), et mettent l'accent en particulier sur deux points : la nécessité d'affirmer le caractère processuel de l'évaluation, l'intérêt d'approches plus participatives.

Derrière ces débats, qu'il s'agisse de textes généraux sur l'évaluation des politiques publiques ou plus spécifiquement de l'évaluation des politiques de clusters, se joue en fait le renoncement à un modèle souvent critiqué, que certains ont qualifié de «balistique » (Padioleau, 1982) ou d' "épidémiologique » (Stame, 2009). Ce modèle voyait dans l'évaluation le dernier maillon d'un processus d'action publique conçu comme séquentiel et linéaire : selon cette conception, la chaîne objectifs-moyens-résultats serait suivie d'une phase de correction, permise par un processus d'évaluation objectif et rigoureux. Mais le modèle qui pourrait s'y substituer se constitue par tâtonnements et les doctrines sont loin d'être stabilisées.

6 Evolution plus spécifiquement anglo-saxonne, qui n'est pas directement transposable au contexte français, pour les raisons évoquées infra. 
En France, selon certains auteurs, on serait davantage actuellement dans un paradigme de l'évaluation «dans l'action » que d'évaluation « de l'action », pour reprendre les termes de Chanut (2009). L'évaluation « dans l'action » fait de l'évaluation un processus quasi-continu, et non spécifiquement ex post. Cette évolution, si elle est avérée, brouille donc les frontières entre évaluation et management ou contrôle de gestion, et contribue à déplacer la charge de l'évaluation des « experts » vers les managers eux-mêmes. Cette assertion est-elle vérifiée dans le cas de l'évaluation des politiques de pôles de compétitivité ? C'est ce que nous examinerons dans la suite, en présentant les modalités de cette évaluation et ses usages. Mais auparavant, nous allons préciser les principales questions que pose en pratique l'évaluation des politiques de clusters.

\section{L'évaluation des politiques de clusters en pratiques}

La multiplication des politiques de clusters, dans un grand nombre de pays, s'explique assez bien : schématiquement, dans un contexte où la mondialisation exacerbe la compétition internationale, et où les pays développés voient avec inquiétude leurs capacités de production, puis de R\&D, délocalisées vers les pays émergents, le constat du succès économique de certains clusters spontanés - dont l'exemple emblématique est la Silicon Valley (Weil, 2010) -, a conduit la puissance publique à tenter de soutenir, de manière volontariste, l'émergence et le développement de clusters (Rosenberg, 2002). Ces politiques, s'appuyant sur un certain nombre de travaux théoriques (cf. revues de littérature dans FenChong, 2009 ou Cruz \& Teixeira 2009), ont pour point commun de consacrer des ressources publiques notables (subventions, incitations fiscales, etc.) au développement de ces clusters.

Le souci de rendre compte de l'utilisation de ces fonds et des effets de ces politiques (accountability), la volonté d'améliorer ces politiques ou de les réorienter, mais aussi des doutes persistants sur les effets des clusters sur la croissance et ses mécanismes (Martin \& Sunley, 2003; Potter \& Miranda, 2009; Torre, 2008) ainsi que sur la capacité des politiques à contribuer à la création et à la dynamique des clusters, sont à l'origine de la multiplication des évaluations commanditées par les pouvoirs publics dans différents pays. La France, après le lancement de sa politique de pôles de compétitivité, n'a pas échappé à la règle. Ce besoin d'évaluation étant posé, qu'évalue-t-on en pratique et comment ${ }^{7}$ ?

\subsection{Que faut-il évaluer? Les objectifs de l'évaluation et la question de l'imputabilité}

Une des difficultés classiques de l'évaluation de politiques publiques est en effet la variété des angles d'attaque qui peuvent se dissimuler sous l'unique vocable « évaluation ». En ce qui concerne les politiques de clusters, différents niveaux d'évaluation, qui renvoient souvent à des acteurs aux intérêts différents, peuvent être distingués :

- d'abord, l'évaluation des politiques de clusters, c'est-à-dire des modalités de mise en œuvre et de l'efficacité de ces politiques, prises au niveau national, par opposition à l'évaluation individuelle des clusters;

7 Nous nous appuyons sur un travail bibliographique comparatif réalisé sur les démarches d'évaluation de politiques de clusters : voir Lefebvre P. \& Pallez F., 2009. Évaluation de la performance des pôles - Approches comparées. Mines ParisTech - Centre de gestion scientifique, rapport commandité par Advancia-Negocia et financé par la Chaire Entreprenariat CCIP. 
- ensuite, si l'on s'intéresse à l'évaluation d'un cluster, on pourra distinguer : l'efficacité de son organisation (structuration, gouvernance, pilotage, etc.), ses résultats par rapport aux objectifs visés (par exemple le nombre de projets collaboratifs qu'il a suscités), son impact sur le territoire et sa dynamique économique (en termes de richesse, d'emplois, d'entreprises créées, etc.), son impact sur les acteurs (entreprises, organismes de recherche, collectivités territoriales).

Ces différents éléments ne sont évidemment pas indépendants, comme l'illustre le schéma ci-dessous qui met bien en évidence la différence entre évaluation d'une politique de clusters et évaluation des clusters eux-mêmes. Malgré son caractère simplificateur, le schéma montre également les déterminants potentiels des résultats atteints par un cluster, qui peuvent être liés à la politique publique en cause, à l'organisation interne du cluster, à des facteurs externes nationaux, mais aussi à des éléments de contexte propres à chaque cluster, comme ses caractéristiques « héritées ». Par ce terme, nous entendons notamment la configuration des acteurs concernés, les ressources et les liens qu'entretiennent entre eux, et avec le territoire, ces différents acteurs, avant même la création institutionnelle du cluster (FenChong, 2009).

Schéma 1 : Niveaux d'évaluation d'un cluster inspiré du BIPE ${ }^{8}$ (2007) et de FenChong (2009)

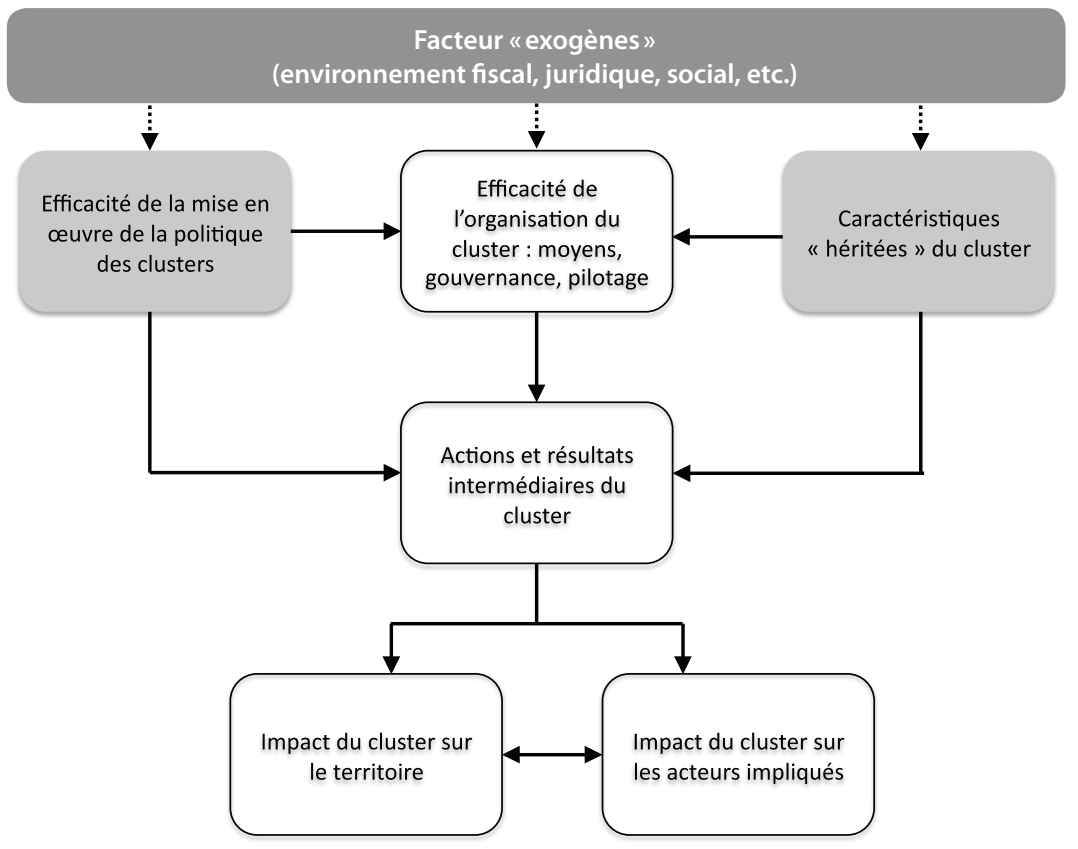

Ces différentes remarques permettent de comprendre la relative variété des démarches d'évaluation des clusters à travers le monde, au-delà d'une diversité liée à des traditions nationales. Ce schéma permet aussi de comprendre que l'évaluation de l'efficacité de l'organisation d'un cluster, sur laquelle il est souvent mis l'accent, n'est qu'une des composantes

8 Cabinet ayant réalisé un benchmark international des démarches d'évaluation de politiques de clusters, préparatoire à l'évaluation des pôles de compétitivité en 2008 (BIPE 2007). 
de l'évaluation d'une politique de cluster. Enfin, il met en évidence le caractère systémique dont nous avons déjà parlé, source d'un problème d'imputabilité des résultats observés à une politique donnée. En effet :

- en théorie, l'évaluation devrait être conduite par rapport à des objectifs définis ex ante, et sur la base d'une comparaison avec une situation de référence elle-même définie et caractérisée avant le lancement de la politique.

- en pratique, on observe en général que les réseaux d'acteurs rassemblés au sein du cluster préexistaient, que la dynamique de développement était déjà en germe, que les objectifs que se fixe le cluster évoluent au fil du temps et que différents facteurs exogènes peuvent avoir joué sur les résultats observés. Ces constats rendent délicate toute évaluation, et notamment tout jugement, en termes d' "additionnalité » de la politique, au sens de la Commission Européenne.

\subsection{Démarche et méthodologie}

\section{Qui commandite l'évaluation et à quel moment?}

Les évaluations sont toujours effectuées à la demande d'un acteur particulier, à un instant donné. Ces deux variables (origine et moment de la demande) influent sur la nature des questions posées, différente selon le type d'acteurs et selon la phase de développement du cluster. En l'occurrence, la plupart des évaluations de politiques de clusters sont faites à l'initiative des acteurs publics concepteurs et financeurs de la politique. En outre, dans le cas français, mais cela est vrai aussi pour beaucoup d'autres pays, l'évaluation est faite dans une phase relativement précoce du développement de la politique (trois ans après son lancement).

\section{Quels indicateurs?}

Les critères pour évaluer les clusters découlent des «objets » que l'on a choisi d'évaluer, eux-mêmes choisis en fonction du moment de l'évaluation et des intérêts du commanditaire. Ces indicateurs concernent en général aussi bien l'organisation du cluster que ses résultats, de toute nature ${ }^{9}$. Par exemple la qualité de l'animation sera jugée par des indicateurs comme le nombre et le coût des actions soutenues. On trouve des indicateurs de résultats « intermédiaires » qui qualifient l'action du cluster comme par exemple le montant consacré aux projets de R\&D ou le montant des investissements réalisés (plateformes de recherche, etc.). La performance scientifique et technique sera estimée par le nombre de brevets et licences. Quant aux impacts « finaux» du cluster, ils sont qualifiés en général par des indicateurs économiques très classiques comme des indicateurs de croissance et de santé des entreprises (chiffre d'affaires, valeur ajoutée, taux d'exportation, etc.), mais également, pour apprécier l'impact sur le territoire, par des indicateurs de création d'emplois, de création d'entreprises, ou d'investissements directs sur ledit territoire.

\subsection{Usages et effets des évaluations}

Sans étude empirique détaillée, il est assez difficile d'appréhender les effets, directs et indirects des évaluations commanditées par les pouvoirs publics et même les usages

\footnotetext{
9 Toutefois, certains pays, comme l'Allemagne, disposant de plus de recul temporel, ont choisi de centrer leur évaluation des programmes BioRegio et Bioprofile sur les impacts économiques sur les territoires (10 ans après le lancement des programmes).
} 
que ceux-ci en font. En effet, les liens entre recommandations des évaluateurs et décision politique n'ont rien de mécanique.

À travers les quelques cas que nous avons pu étudier à l'étranger, et qui ne seront pas développés ici (Gallié, Glaser, \& Pallez, 2010, 2011), nous nous contenterons de noter que les différentes évaluations ont toutes conduit à une reconduction des politiques de cluster, en suggérant des améliorations des dispositifs dans différents domaines (pilotage, financement...). Dans tous les cas, l'évaluation est considérée comme un outil pour réfléchir à la suite de la politique, et non comme un couperet qui déterminerait à lui seul de la poursuite ou non de la politique. Quant à l'évaluation des clusters eux-mêmes, elle a pu, dans certains cas, conduire à des arrêts du soutien public, comme par exemple dans la région wallonne (arrêt du financement de deux clusters) (cf. Lepage, 2009) ou en France (délabellisation de six pôles de compétitivité).

Après avoir donné cet aperçu général des pratiques d'évaluation des politiques de clusters, nous allons présenter plus en détail un cas particulier, le cas français.

\section{L'évaluation, composante de la politique française des pôles de compétitivité}

En France, la politique des pôles, par contraste avec nombre de politiques antérieures, s'est construite en intégrant dès l'origine la question de l'évaluation. Dès le lancement de la politique des pôles, l'État avait prévu de l'évaluer au bout de trois ans et d'utiliser les résultats pour réorienter éventuellement la doctrine initiale. Sans être totalement nouvelle ${ }^{10}$, cette particularité est suffisamment notable pour que nous la signalions. Naturellement nous distinguons cette démarche d'évaluation institutionnelle des travaux de chercheurs, qui, selon des méthodes diverses, se sont intéressés à la performance des pôles français (voir par exemple Chalaye \& Massard, 2009; Duranton, Martin, Mayer \& Mayneris, 2008; Bocquet \& Mothe, 2009).

Pour présenter les caractéristiques de la démarche d'évaluation menée en France, nous procéderons en deux temps :

- dans un premier temps, nous décrirons factuellement la conception de la démarche en reprenant les rubriques générales que nous venons de présenter succinctement : objectifs et type de résultats évalués, méthodologie et démarche adoptées. Nous commenterons brièvement au passage les choix qui ont été faits par rapport aux questionnements généraux évoqués ci-avant;

- puis nous analyserons plus en détail la manière dont cette évaluation a été utilisée, et montrerons qu'elle est conçue comme un outil de pilotage.

Nous nous appuierons pour ce faire sur un matériau constitué :

- des textes publics de synthèse produits par les évaluateurs en 2008, mais aussi d'un certain nombre de rapports d'évaluation confidentiels fournis par des pôles avec qui nous étions en contact;

- des séances de l'Observatoire des Pôles de Compétitivité, séminaire mensuel au cours duquel dialoguent des chercheurs et des acteurs impliqués dans la politique des pôles (dirigeants de pôles, représentants de l'État et des collectivités territoriales, responsables

${ }^{10}$ On se rappelle que, dès 1988, le lancement du RMI s'était accompagné d'un engagement de procéder à une évaluation. 
d'entreprises ou d'établissements d'enseignement supérieur et de recherche, consultants...). L'une de ces séances, notamment, a été consacrée à l'évaluation de 2008;

- d'entretiens avec les évaluateurs ainsi qu'avec divers responsables de pôles (une quinzaine) et des représentants de l'Administration (DGCIS), pendant et après la phase d'évaluation.

\subsection{La démarche d'évaluation des pôles : objectifs, méthodes, résultats}

Après avoir établi un cahier des charges s'appuyant sur le cadrage fourni par le BIPE, la DIACT (Délégation Interministérielle à l'Aménagement et à la Compétitivité des Territoires) ${ }^{11}$ lança un appel d'offres en 2007, qui fut remporté par deux cabinets de conseil, CM International (CMI) et Boston Consulting Group (BCG). Sur la base du cahier des charges, les cabinets précisèrent les objectifs et la méthodologie.

\section{Les objectifs de l'évaluation}

La mission consistait à mener de front l'analyse de l'orientation stratégique et des modalités d'intervention de la politique nationale et celle de la cohérence et de l'efficacité du dispositif, pôle par pôle, donc de manière exhaustive. On notera en cela l'ambition de la démarche, mais aussi l'idée, pertinente, de notre point de vue, que l'évaluation de la politique nationale devait s'alimenter de la connaissance fine des dispositifs qu'elle avait créés, à savoir les pôles.

L'évaluation de la politique s'intéressait à trois grands thèmes : sa pertinence/cohérence, sa mise en œuvre et ses premiers effets ; les évaluateurs avaient en effet jugé peu pertinent, compte tenu de la jeunesse de la politique, de détecter des impacts économiques. Quant à l'évaluation des 71 pôles, structures légères de statut associatif, dotées de quelques permanents financés par des fonds publics, elle portait surtout sur l'efficacité de ces organisations, et sur leurs premières actions. Elle reposait donc sur l'analyse de trois axes : la dynamique des pôles, leur structuration et leurs projets de R\&D. Sur chaque axe ont été définis les résultats qui seraient considérés comme satisfaisants, moyens ou insuffisants.

Par rapport au schéma 1, on en restait donc à l'évaluation de l'organisation (de sa dynamique) et de résultats intermédiaires (comme les financements obtenus) à l'exclusion de la mesure de tout « impact » final. Quant à la délicate question de l'imputabilité de la politique, elle n'était pas traitée explicitement, les évaluateurs ayant probablement conscience de sa difficulté dans un contexte aussi systémique. En effet, la causalité supposée $R \& D \rightarrow$ innovation $\rightarrow$ compétitivité, qui est à la base de la politique des pôles, n'est pas déterministe. Ainsi des projets de recherche collaboratifs peuvent avoir des impacts très divers, parfois immédiatement visibles s'ils débouchent sur une innovation rencontrant un succès commercial, parfois plus indirects s'ils contribuent seulement à la constitution d'un réseau entre acteurs complémentaires. En outre, il peut s'agir de coalitions opportunistes d'acteurs désireux de trouver des financements complémentaires pour des actions qu'ils souhaitaient engager de toute façon. En France, les évaluateurs ont d'ailleurs remarqué, dans les débuts de la vie des pôles, un effet de « déstockage » de projets de R\&D existants, qui ont pu trouver ainsi un financement.

On notera toutefois que l'évaluation a cherché à analyser la « vie antérieure » des pôles et a donc pris en compte, de manière implicite, le fait que les caractéristiques « héritées » d'un pôle (la nature des partenaires et leurs liens antérieurs, leurs modalités historiques de coopération et d'innovation, etc.) pouvaient expliquer une partie des résultats constatés.

\footnotetext{
${ }^{11}$ Qui a repris, depuis, le nom de DATAR.
} 


\section{La méthodologie et le pilotage de l'évaluation}

La méthodologie utilisait l'analyse documentaire, les entretiens et réunions avec les acteurs et organismes concernés, ainsi qu'une enquête qualitative et quantitative par questionnaire envoyé aux pôles préalablement aux entretiens, ceux-ci étant réalisés selon une procédure formalisée pour garantir la comparabilité et le caractère normé de l'évaluation. Au total, plus de mille personnes furent interrogées, dont 720 au niveau des pôles, soit dix par pôle en moyenne. Une phase de test sur quatre pôles « pilotes » avait par ailleurs permis de mettre au point le questionnaire, de manière à ce qu'il prenne en compte au mieux la diversité des pôles. L'opération fut pilotée de manière rapprochée par les commanditaires : une rencontre par semaine avec la DIACT, des contacts fréquents également avec la Direction générale des Entreprises, un comité interministériel par mois, un comité de pilotage tous les deux ou trois mois... Les résultats globaux de l'évaluation furent présentés au comité de pilotage en juin 2008, et furent suivis d'une communication gouvernementale. Mais seuls les documents de synthèse furent publiés sur le Web, les évaluations « individuelles » des pôles restant strictement confidentielles. Des retours furent par ailleurs effectués vers chacun des pôles classés en $3^{\mathrm{e}}$ catégorie par les évaluateurs (voir supra), sous forme d' "entretiens contradictoires » en présence des différentes parties prenantes (tutelle, gouvernance des pôles, évaluateurs, collectivités territoriales concernées, etc.).

S'appuyant aussi bien sur des analyses qualitatives que quantitatives, l'évaluation a donc utilisé différentes approches méthodologiques, dans un cadre néanmoins très formalisé, qui assurait en particulier une «égalité de traitement» entre les pôles. Mais, même si la démarche semblait emprunter certains de ses traits à l'audit, il s'agissait bien d'explorer des fonctionnements et des dynamiques non prédéterminés par des standards (Fouquet, 2009). En revanche, il ne s'agit pas, à l'évidence, d'une évaluation participative, les commanditaires tenant à maîtriser le processus et ses résultats de bout en bout.

\section{Les résultats de l'évaluation}

La conclusion principale de CMI et BCG fut que le « dispositif des pôles de compétitivité semble suffisamment prometteur pour être maintenu dans ses grands principes ». Elle était assortie de diverses recommandations d'amélioration, sur lesquelles nous reviendrons.

Quant à l'évaluation des pôles de compétitivité, pris isolément, elle conduisit à un classement en trois catégories, proposé par les évaluateurs sur la base de trois axes-clés (stratégie du pôle, gouvernance, capacité à monter des projets de R\&D) :

- les pôles (39) qui « ont atteint les objectifs de la politique des pôles »;

- les pôles (19) qui « ont atteint partiellement les objectifs de la politique des pôles et qui doivent travailler à l'amélioration de certaines dimensions de leur action »;

- les pôles (13) qui « pourraient tirer parti d'une reconfiguration en profondeur ».

Mais, au-delà de ce classement, l'évaluation permit aussi le renvoi à chaque pôle - c'està-dire à sa gouvernance - d'une image de son fonctionnement, à travers une « fiche » assez fouillée d'une quinzaine de pages.

À la suite de ces conclusions, le gouvernement annonça un certain nombre de décisions ${ }^{12}$ qui constituent ce que l'on a appelé « la politique 2.0 » des pôles, et sur lesquelles nous

\footnotetext{
${ }^{12}$ Pour une synthèse, on pourra se reporter au compte rendu de la séance du 21 octobre 2008 du séminaire de l'Observatoire des pôles de compétitivité (http://observatoirepc.org/) auquel participèrent le CMI, le BCG et la DIACT.
} 
reviendrons ci-dessous. D'ores et déjà, notons le lien fort qui a été fait entre les résultats de l'évaluation et l'annonce de la deuxième phase de la politique.

\section{Quel usage a-t-on fait de l'évaluation?}

Il est souvent reproché à l'État français de peu évaluer ses politiques et dans tous les cas, de donner peu de suite à ses évaluations (Duranton, Martin, Mayer \& Mayneris, 2008). Quels ont donc été les effets et les usages de l'évaluation par les différentes parties prenantes? Nous nous interrogeons d'abord sur l'appropriation des résultats de l'évaluation par l'État, puis sur les effets possibles de cette dernière sur les pôles, pour terminer par les apprentissages pour l'évaluation suivante.

\subsection{Une évaluation pour réorienter la politique et améliorer le pilotage}

Nous avons déjà noté que cette démarche évaluative s'inscrit dans un mouvement très général, qui fait de l'obligation de « rendre compte » une exigence maintenant naturelle de la vie économique (Dumez, 2008) et, quand il s'agit de politiques publiques, de la vie démocratique. Cela dit, même si la communication politique sur les résultats de la politique des pôles en était évidemment un objectif important, la démarche d'évaluation était aussi explicitement conçue comme un élément du dispositif de pilotage. Nous allons indiquer comment cela s'est traduit en pratique.

Le principe de la reconduction de la politique des pôles avait été annoncé par le chef de l'État dès juin 2007, soit un an plus tôt. Néanmoins, l'évaluation a permis de montrer la pertinence du dispositif en termes de structuration des acteurs et de réflexion stratégique territoriale, et a conduit l'État à réaffirmer la prorogation de sa politique sur les trois ans à suivre (actuellement prolongée jusqu'en 2012), en renouvelant le budget initial dans les mêmes conditions à savoir 1,5 milliard d'euros.

Plus que la décision même de reconduire la politique nationale des pôles de compétitivité, que l'on ne peut imputer à l'évaluation, la nouvelle politique 2.0 peut lui être reliée. Il y a eu, bien sûr, avant l'évaluation, de nombreuses occasions d'interactions entre les pôles et les représentants de leur tutelle. Mais il est indéniable que certains des nouveaux dispositifs exploitent directement les résultats et puisent dans les nombreuses préconisations issues de l'évaluation. Nous commentons ci-dessous deux de ces dispositifs qui nous semblent parmi les plus significatifs.

Une formalisation accrue de la stratégie

Ainsi, l'évaluation met en avant la faiblesse des pôles en matière d'élaboration et de formalisation de leur stratégie. Il semble que la conséquence de ce résultat a été, à la demande de l'État, la rédaction de « feuilles de route technologiques » par les pôles et la signature d'un « contrat de performance » pluriannuel entre l'État, les collectivités territoriales et chaque pôle. Dans ce contrat, signé par le président du pôle, celui-ci s'engage sur un programme d'actions, assorti d'un calendrier de réalisation. La tutelle montre donc un désir de formalisation accrue de la relation entre l'État et les pôles, avec des objectifs pour chaque pôle mieux définis.

La mise en place d'indicateurs de suivi des résultats

Dans la logique du contrat de performance, la tutelle impose maintenant à chaque pôle la mise en place d'indicateurs. L'insistance sur la construction d'indicateurs est particulièrement intéressante, car elle montre dès à présent la préoccupation de l'État pour l'évaluation 
future de l'action du pôle. Une première série d'environ 70 indicateurs sont communs à tous (création d'entreprises, nombre de projets de R\&D retenus pour un financement public, nombre de brevets, etc.). Ces indicateurs doivent faciliter la comparaison entre les pôles. Mais l'État a également pris en compte la diversité des pôles, en demandant à chacun de produire des indicateurs spécifiques.

Même si, en apparence, ces évolutions paraissent relativement attendues, on peut y déceler des éléments plus originaux, que l'on peut interpréter comme le résultat d'un apprentissage de la tutelle, obtenu notamment à travers le processus d'évaluation. Ce nouveau savoir pourrait être sommairement qualifié comme suit : affirmation du lien entre la dynamique de développement et la formulation détaillée d'une stratégie (y compris en termes technologiques), que les pôles sont dès lors encouragés à établir par un formalisme contractuel; reconnaissance de l'existence de schémas de développement diversifiés des pôles, au-delà d'un socle d'indicateurs communs à tous.

\subsection{Une évaluation pour mobiliser les pôles}

L'évaluation peut aussi avoir des effets directs sur les pôles. En effet, à travers la connaissance « pôle par pôle » qu'elle apporte, les comparaisons qu'elle autorise, et surtout, la légitimité qu'apporte une expertise extérieure aux décisions étatiques, l'évaluation peut permettre de piloter ou de mobiliser les pôles, voire de les sanctionner. Il est intéressant d'analyser les choix de l'État en la matière.

L'effet le plus visible de l'évaluation pour les pôles a été leur classement en trois catégories. Un tel classement récompense symboliquement les «bons élèves ». Mais les 13 pôles classés en catégorie 3 l'ont vécu comme une sanction. Certains des pôles mal classés se sont interrogés sur la pertinence même des critères utilisés. On peut en effet défendre l'idée que la compétitivité de certains pôles reposerait moins sur des innovations technologiques radicales que sur des innovations par l'usage (exemple du pôle Enfant, construit autour d'un marché13); ou sur la disponibilité d'une main-d'œuvre formée à des compétences spécialisées et l'organisation d'une filière industrielle (exemple du Pôle Nucléaire de Bourgogne (Fen Chong \& Pallez, 2008)). Or ces critères ont été peu pris en compte par les évaluateurs. Ces pôles ont pu alors considérer qu'ils n'avaient pas été évalués à l'aune de leurs spécificités.

Pour la tutelle, diverses attitudes étaient envisageables à l'issue de l'évaluation : sanctionner immédiatement en délabellisant les « mauvais » pôles pour se focaliser sur les plus performants, se rapprochant ainsi d'un schéma plus « élitiste » que certains avaient défendu lors du lancement de la politique; ou conserver pour l'instant le statu quo, en espérant que la remobilisation soit forte d'elle-même.

L'État a opté pour une position intermédiaire, en proposant aux pôles mal classés une phase transitoire de réorganisation/restructuration, qui devait durer un an, mais s'est en fait prolongée presque deux ans. Des décisions de délabellisation, qui ont touché finalement six des treize pôles classés en catégorie 3, n'ont en effet été prises qu'en mai 2010.

Cette position a eu des effets notables puisque l'on a pu observer une motivation certaine des pôles mal classés à travailler sur des réorientations de leur stratégie et de leur

${ }^{13}$ Voir l'audition du Pôle Enfant par la Mission d'évaluation et de contrôle de l'Assemblée Nationale : http://www.assemblee-nationale.fr/13/cr-mec/08-09/c0809045.asp 
fonctionnement. En outre, l'électrochoc lié à l'évaluation a conduit à une forte mobilisation des partenaires territoriaux de ces pôles en leur faveur, parfois à un haut niveau politique (députés, sénateurs, présidents de conseils régionaux, dont certains anciens ministres). Cette mobilisation était évidemment liée à la menace crédible d'une délabellisation qui constituait une sanction symbolique lourde, risquant de plus d'entraîner des conséquences importantes sur le pôle (perte de visibilité, tarissement de certains financements publics, etc.) et sur son territoire (risques accrus de délocalisation, démobilisation des acteurs privés, recentrage des chercheurs sur une recherche plus académique, etc.). On notera que le poids important accordé aux « labels » est probablement une caractéristique profonde de la culture nationale française, attachée aux marques d'excellence attribuées par l'État.

Ce faisant, la tutelle a utilisé l'effet de sunshine regulation, jouant sur la publication (et donc la comparaison) des résultats. Mais elle l'assortissait aussi d'une procédure plus directive : injonction pour certains pôles à la « remise en ordre » et deuxième évaluation (effectuée pendant le deuxième semestre 2009 par les mêmes évaluateurs et avec la même grille que la première évaluation), avec une échéance et une "menace ». Plus généralement, même pour les pôles mieux classés, le regard extérieur porté par des évaluateurs et la publicité donnée aux résultats ont constitué un incitatif à la réflexion interne et à la remise en cause de certains fonctionnements. D'autant que l'évaluation de 2012 s'appuiera nécessairement sur les résultats de l'évaluation de 2008.

\subsection{Des pistes d'apprentissage pour une évaluation future?}

Peut-on aussi tirer de cette expérience des enseignements pour la prochaine évaluation? Il nous semble que l'expérience qui vient de se dérouler en France peut modifier sensiblement les choses sur deux points : l'objectivation des jugements portés par les évaluateurs et la prise en compte de la diversité des pôles.

\section{L'objectivation des jugements}

En matière d'objectivation des données et de rigueur de la méthode, l'élaboration des feuilles de routes et des contrats de performance devrait contribuer à faciliter la prochaine évaluation puisque les objectifs auront été plus clairement formulés, et que des indicateurs normalisés ont été fixés. En outre, grâce à la première évaluation, une situation de référence aura été définie, permettant ainsi d'objectiver la notion de trajectoire.

Quant à la question de la fiabilité des données, on pourrait craindre qu'elle reste sujette à caution, les évalués étant pour partie les fournisseurs de ces données. Même si le risque n'est pas nul, il nous semble qu'il doit être mis en balance des cercles vertueux enclenchés dans les pôles par cette obligation (incitation à l'autoévaluation). En outre, la confrontation de données quantitatives aux points de vue des acteurs, qui du coup devrait être systématisée par la tutelle pour permettre de contrôler la fiabilité, peut être en soi source de connaissance, notamment pour mettre en évidence les schémas de causalité expliquant la performance. Mais, ce faisant, on accepte une conception moins positiviste de la vérité, en se contentant de vérités « non invraisemblables » (GRETU, 1980).

Une meilleure prise en compte de la diversité des pôles?

Par ailleurs, on l'a dit, un des enjeux futurs réside dans la prise en compte de la diversité des pôles dans les évaluations à venir. Il s'agirait en effet de mener les compa- 
raisons au sein de catégories au sein desquelles la comparabilité entre pôles, en termes de trajectoires de développement et de performance attendue, soit avérée. Mais sur quelles bases construire cette typologie?

Les différences sectorielles sont classiquement une première explication pour justifier la diversité. Par exemple Colgan et Baker (2003) proposent de regrouper les clusters du Maine, qu'ils étudient, en trois groupes. Cette classification, basée sur la nature des ressources mobilisées (technologie, ressources naturelles, autres), doit permettre, selon eux, de différencier les clusters, et donc d'en améliorer le pilotage par les autorités publiques. Mais la richesse des données rassemblées lors de la première évaluation par le CMI et le BCG, et de celles que les différents organismes publics concernés accumulent actuellement, permet maintenant de dépasser ces typologies simples et intuitives (Bonnafous-Boucher \& Saussois, 2010; Caillou et al., 2012; Glaser, Gallié, Mérindol, \& Weil, 2010 ; Hussler, Muller, \& Ronde, 2010). Sur cette base, on pourra ainsi travailler simultanément sur la question de l'imputabilité des résultats et sur la différenciation des trajectoires et des performances des pôles.

À travers ce passage en revue des effets de l'évaluation, on retrouve les grandes catégories d'usages déjà identifiées dans la littérature : aide à la décision et au pilotage, mobilisation des acteurs et construction de visions partagées, apprentissage (tutelle autant que gouvernance des pôles). Si l'effet direct de l'évaluation (au sens de ses résultats finaux) sur la reconfiguration de la politique a été relativement modéré, on peut en revanche faire l'hypothèse que le processus même de l'évaluation a fait mûrir certaines idées (par exemple élargissement des missions des pôles, ou lancement d'une politique de « grappes » d'entreprises ${ }^{14}$ ) et a contribué à un processus réflexif pour tous les acteurs concernés.

\section{Conclusion}

Cette exploration de l'évaluation des pôles de compétitivité nous incite, en conclusion, à nous demander comment caractériser le modèle d'évaluation qui ressort de cette expérience et s'il est par ailleurs conforté par d'autres exemples dans d'autres secteurs.

Sur le premier point, il nous semble que la démarche que nous avons analysée, critiquable à certains égards, porte néanmoins en germe un nouveau modèle de l'évaluation,

- qui abandonne l'ambition d'être la seule source des décisions stratégiques;

- qui renonce à une vision positiviste en laissant place à des approches plus qualitatives,

- qui, par la mobilisation qu'elle suscite, accélère la construction des collectifs et des référentiels communs que la politique des pôles elle-même souhaitait encourager;

- qui, finalement, par les apprentissages qu'elle induit, participe à la production simultanée d'une politique publique et de sa doctrine de pilotage.

Ces différents éléments nous semblent en faire une démarche qui rejoint le modèle de l'évaluation "dans l'action». Une évaluation «de l'action» (ou ex post) viserait en effet à mesurer les résultats des actions au regard des objectifs d'une politique parvenue à maturité afin d'en tirer les enseignements rétrospectifs. Or, étant donné la temporalité du processus de développement, cette conception n'est pas soutenable. Et pourtant, compte tenu des sommes allouées, l'évaluation à trois ans résulte d'une exigence démocratique,

${ }^{14}$ Des grappes d'entreprises sont des clusters d'orientation moins technologiques et plus petits que les pôles. 
qui oblige à s'interroger sur la pertinence et la réorientation au plus juste de la politique. Le schéma de l'évaluation «dans l'action » tel que nous avons essayé de le caractériser, permet de dépasser cette contradiction.

Retrouve-t-on ces caractéristiques dans les dernières évaluations de politiques publiques réalisées en France ? Répondre à cette question nécessiterait une analyse systématique qui dépasse le propos de cet article. Mais sur l'exemple de l'évaluation récemment réalisée par la Cour des Comptes sur la politique éducative on peut déjà remarquer que, malgré les nombreuses différences des deux démarches, il existe un point commun dans la philosophie de l'évaluation, telle qu'elle est présentée par Jean Picq, président de la troisième chambre de la Cour des comptes : la volonté d'ouvrir des questionnements plus que de clore les débats par des réponses, l'exigence d'humilité qui découle des imperfections de toute évaluation, l'évaluation vue comme élément d'un processus qui se déroule sur le temps long.

Pour conclure, il faut prendre conscience que ce positionnement de l'évaluation comme une des composantes du dispositif de pilotage, lui confère en même temps un statut plus modeste : l'évaluation devient un outil «parmi d'autres », autant à la disposition des responsables qui conduisent une politique, que des acteurs évalués. Elle s'éloigne de ce fait d'un modèle de production de connaissance objective, incontestable, indépendante, dictant ses décisions au décideur, modèle qui a longtemps constitué l'ultima ratio de l'évaluation. Une évaluation plus modeste mais plus utile,n'est-ce pas la voie de l'évaluation moderne? 


\section{Bibliographie}

BACHTLER J., \& WREN C., (2006). Evaluation of European Union Cohesion Policy: Research Questions and Policy Challenges, Regional Studies 40 (2), 143-153.

BELLANDI M., \& CALOFFI A., (2010). Towards a framework for the evaluation of policies of cluster upgrading and innovation, Revue d'économie industrielle 129-130, 259-276.

BIPE, (2007). Méthodes et outils d'évaluation des clusters appliqués aux pôles de compétitivité. BIPE, Paris.

BOCQUET R., MOTHE C., (2009). Gouvernance et performance des pôles de PME, Revue Française de Gestion 190, 101-122.

BONNAFOUS-BOUCHER M. et SAUSSOIS J.-M. (dir.), (2010). La dynamique entrepreneuriale dans les pôles de compétitivité, Prospective et Entreprise, CCIP (Advancia-Negocia, ESCP Europe), Paris.

CAILlOU P., GALLIÉ E.-P. et al., (2012). Typologie des pôles de compétitivité basée sur leurs caractéristiques « héritées », DATAR, Paris, Travaux en ligne, http://www.territoires.gouv.fr/travaux-en-ligne

CHALAYE S. et MASSARD N., (2009). Les clusters : diversité des pratiques et mesures de performance, Revue d'économie industrielle 128, 153-176.

CHANUT V., (2009). Pour une nouvelle geste évaluative. In S. Trosa (Ed.), Évaluer les politiques publiques pour améliorer l'action publique - Une perspective internationale, Comité pour l'histoire économique et financière de la France, Paris.

CRUZ S. C. S. et TEIXEIRA A. A. C., (2009). The Evolution of the Cluster Literature: Shedding Light on the Regional Studies-Regional Science Debate, Regional Studies 44 (9), 1263-1288.

DIEZ A. M., (2001). The Evaluation of Regional Innovation and Cluster Policies: Towards a participatory approach, European Planning Studies 9 (7), 907-923.

DUMEZ H. (2008). Coordination du dossier : L'obligation de rendre des comptes, Gérer et Comprendre 91, 4-54.

DURANTON G., MARTIN P., MAYER T. et MAYNERIS F., (2008). Les pôles de compétitivité : que peut-on en attendre? Opuscules du CEPREMAP, Ed. ENS, Paris.

FEN CHONG S. et PALlEZ F., (2008). Le Pôle Nucléaire de Bourgogne, ou l'art du décalage, Réalités industrielles, mai.

FEN CHONG S., (2009). Le pilotage chemin faisant- Émergence des modes de gouvernance et de pilotage des pôles de compétitivité, Thèse de doctorat en sciences de gestion, Paris IX Dauphine.

FETTERMAN D., KAFTARIAN S. J. et WANDERMANS A. (Eds.), (1995). Empowerment Evaluation: Knowledge and Tools for Self-assessment and Accountability, Sage Publications, Thousand Oak.

FOUQUET A., (2009). L'évaluation, concepts et enjeux. In S. Trosa (Ed.), Évaluer les politiques publiques pour améliorer l'action publique - Une perspective internationale, Comité pour l'histoire économique et financière de la France, Paris.

GALLIÉ E.-P., GLASER A. et PALLEZ F., (2010). Public Policy and Evaluation, a New Process in Construction - The Case of the French Cluster Evaluation, Regional Science Association International - British and Irish Section Conference, Glasgow.

GALLIÉ E.-P., GLASER, A. et PALLEZ F., (2011). Évaluer la politique des pôles de compétitivité : quels principes, quels usages? Observatoire des pôles de compétitivité, Paris.

GIBERT P., (2003). L'évaluation de politique : contrôle externe de la gestion publique? Revue Française de Gestion 147, 258-273.

GLASER A., GALLIÉ E.-P., MÉRINDOL V. et WEIL T., (2010). The Link between a Cluster's Governance and its Capability to get Collaborative Research Projects funded: An investigation of French clusters, $4^{\mathrm{e}}$ Atelier "Stratégies, Espaces et Territoires", Toulouse.

GRETU (Groupe de réflexion sur 1'économie des transports urbains), (1980). Une étude économique a montré... - Mythes et réalités des études de transport, Éditions Cujas, Paris.

GUBA E. G. et LINCOLN Y. S., (1989). Fourth Generation Evaluation, Sage Publications, Thousand Oaks. 
HUSSLER C., MULLER P. et RONDE P., (2010). Les pôles de compétitivité : morphologies et performances, European Localized Innovation Observatory (EuroLIO) Conference, Toulouse.

LACASSE F., (1995). Mythes, savoirs et décisions politiques, PUF, Paris.

LEFEBVRE P. et PALLEZ F., (2009). Évaluation de la performance des pôles - Approches comparées. Paris, Mines ParisTech - Centre de gestion scientifique.

LEPAGE V., (2009). L'évaluation externe comme outil de pilotage de la politique de clustering : Leçons tirées de 5 années de pratique en région Walonne, Conférence "Pôles de compétitivité et développement économique régional", Liège.

MARTIN R. et SUNLEY P. (2003). Deconstructing Clusters: Chaotic Concept or Policy Panacea? Journal of Economic Geography 3 (1), 5-35.

MENU S., (2011). Les Pôles de Compétitivité, un nouveau pilotage de la politique industrielle ? Bilan en Ile-deFrance, Politiques et Management Public 28 (1), 105-121.

PADIOLEAU J.-G., (1982). L'État au concret, PUF, Paris.

PAWSON R., (2006). Evidence based Policy: the realist synthesis, Sage, Londres.

PERRET B., (2001). L'évaluation des politiques publiques, La Découverte, Paris.

PERRET B., (2008). L'évaluation des politiques publiques - Entre culture du résultat et apprentissage collectif, Esprit 350, 142-159.

POTTER, J., \& MIRANDA, G. (Eds.). (2009). Pôles de compétitivité, innovation et entrepreneuriat, Paris, OCDE.

POTTER P., (2005). Facilitating Transferable Learning through Cluster Evaluation: New Opportunities in the Development Partnerships of the EU 'EQUAL’ Programme. Evaluation 11 (2), 189-205.

ROSENBERG D., (2002). Cloning Silicon Valley: The Next Generation High Tech Hotspots, Pearson Education Ltd, London.

RUSSO M. et ROSSI F., (2009). Cooperation Networks and Innovation: A Complex Systems Perspective to the Analysis and Evaluation of Regional Innovation Policy Programme, Evaluation 15 (1), 75-99.

SÖLVELL Ö., (2009). Clusters - Balancing evolutionary and Constructive Forces, Ivory Tower Publishers, Stockholm.

STAME N., (2009). Avantages et inconvénients des différentes méthodes d'évaluation : comment choisir? In S. Trosa (Ed.), Évaluer les politiques publiques pour améliorer l'action publique - Une perspective internationale, Comité pour l'histoire économique et financière de la France, Paris.

THOENIG J. C., (2002). L'évaluation en actes : leçons et perspectives, Politiques et management public 20 (4), $33-50$. 